\title{
A Review of HIV Pre-exposure Prophylaxis Streamlining Strategies
}

\author{
Aaron J. Siegler ${ }^{1}$ (D) $\cdot$ Kevin Steehler ${ }^{1,2} \cdot$ Jessica M. Sales ${ }^{1} \cdot$ Douglas S. Krakower $^{3}$
}

Published online: 13 September 2020

(C) The Author(s) 2020

\begin{abstract}
Purpose of Review Standard care for HIV pre-exposure prophylaxis (PrEP) in the USA creates substantial burdens for patients, clinicians, and the healthcare system; to optimize uptake, there is a need for innovative strategies to streamline its provision. Recent Findings Our review, structured by the expanded chronic care model, identified eleven promising strategies to streamline PrEP care. Approaches ranged widely in mechanism of action. Using text messages to support care was the only strategy with clinical trial evidence supporting its use. Other modalities such as patient navigation, telemedicine PrEP models, alternate dosing availability, same-day prescription, and provider training have promising pilot or associational data and seem likely to lower barriers to entering into or remaining in care. Many of the strategies have established success in related domains such as HIV care, meriting consideration in evaluating their use for PrEP.

Summary Making PrEP care less burdensome will be an important part of bringing it to scale. Text message interventions have proven efficacy and merit broad adoption. Encouraging preliminary evidence for other strategies indicates the importance of building a stronger evidence base to clarify the effect of each strategy. Ongoing development of an evidence base should not delay the use of these promising strategies; instead, it calls for careful consideration for how each program may best match its environment to facilitate PrEP prescribing and use.
\end{abstract}

Keywords Chronic care model $\cdot$ mHealth $\cdot$ Telemedicine $\cdot$ PrEP

\section{Introduction}

The first major trial of HIV pre-exposure prophylaxis (PrEP), iPrEX, demonstrated the high efficacy of PrEP in 2010 [1]. By 2018, an estimated 380,000 persons had initiated PrEP globally, with the majority $(225,000)$ residing in the USA [2]. In New South Wales, Australia, an encouraging PrEP implementation project initiated 9700 PrEP users across 21 sites. A populationlevel decline in new HIV diagnoses was observed after the first phase of the project, with 295 new diagnoses decreasing to 221 new diagnoses, a 25\% decline. Similarly, in San Francisco, as

This article is part of the Topical Collection on The Science of Prevention

Aaron J. Siegler

asiegle@emory.edu

1 Department of Behavioral Sciences and Health Education, Emory University, Rollins School of Public Health, Atlanta, GA, USA

2 Emory University, School of Medicine, Atlanta, GA 30322, USA

3 Division of Infectious Diseases, Beth Israel Deaconess Medical Center, The Fenway Institute, Fenway Health, Department of Population Medicine, Harvard Medical School, Boston, MA, USA
PrEP and other "getting to zero" efforts scaled up rapidly, new HIV diagnoses were observed to decline substantially [3]. To observe similar population-level gains elsewhere, it will be necessary to bring PrEP to scale for groups with high HIV incidence. Scaling up PrEP to optimal levels will require a considerable investment and effort. UNAIDS has set a goal of having 3 million PrEP users by 2020, and current progress is one-tenth of that level.

PrEP is a biobehavioral intervention requiring clinician oversight of care. Nearly all guidance for PrEP care, including from the World Health Organization and from the US Centers for Disease Control and Prevention [4, 5], places substantial responsibilities on both individuals seeking PrEP and healthcare systems providing it. For individuals, seeking care typically comprises four clinical visits per year with laboratory testing, counseling as needed, and renewed prescriptions. For healthcare systems, PrEP visits require both clinician time and physical resources to host the visit, including laboratory services and supplies. The overall model of PrEP care therefore requires training providers to prescribe PrEP, developing systems to support the financing of PrEP medication, and supporting the quarterly monitoring of individuals over time to ensure they can remain on PrEP. 
In order to expand access to the millions in need, it will be important to ease burdens for PrEP users, prescribing clinicians, and healthcare systems. In this article, we review advances to date in efforts to streamline PrEP care, with a focus on the healthcare system of the USA.

\section{Expanded Chronic Care Model}

We leverage components of the expanded chronic care model (ECCM) to structure our review of opportunities for streamlining PrEP care, focusing on interventions that decrease burdens borne by patients and healthcare systems (Fig. 1). The chronic care model was developed to facilitate the implementation of evidence-based methods into disease management practices and has since been expanded to incorporate prevention practice to allow for its integration into public health [6]. ECCM has been widely used and has a strong base of evidence with both randomized controlled trials and implementation science designs indicating efficacy across a variety of health conditions [7]. We selected ECCM domains that align with our review of current evidence regarding simplified PrEP care [6, 8]. For clinicians and community members to optimize decisions to engage in care (decision support), they need a lower burden of care (streamlined delivery approaches), self-efficacy to engage and continue in care (patient skill management), systems to support their preventive health choices (improved health information systems), and healthy public policies to support all components of the model. We review interventions in each area, describing current research and future opportunities.

\section{Facilitate Patient Management Support}

\section{mHealth Solutions}

There are numerous mHealth-based PrEP adherence and retention support interventions. At their most basic, interventions provide electronic reminders to take daily PrEP through means such as electronic text messages (SMS) or in-app notifications. Weekly text message reminder systems have been demonstrated in international settings to improve adherence to HIV medication regimens [9]. The proliferation of free pill reminder smartphone apps, as well as native, customizable reminder alerts/alarms in smartphone operating systems, makes such trials harder to assess in settings with higher smartphone ownership.

Two trials among MSM in the USA sought to increase PrEP adherence through enhanced messaging formats. The TAPIR trial assessed provision of automated, personalized daily PrEP text message reminders, finding no improvement in the primary outcome of protective levels of PrEP as determined by levels of tenofovir-diphosphate (TFV-DP) indicative of $\geq 4$ days per week dosing [10]. A secondary analysis found a TFV-DP threshold indicative of 7 days per week dosing that was higher in the intervention than the control condition, signaling that the intervention may have had a larger impact on a population with lower overall PrEP adherence. The EPIC trial assessed provision of an interactive text message platform with weekly "check-in" messages from study counselors and optional automated daily PrEP reminders $[11 \bullet \cdot]$. This intervention had a significant impact on retention in care with $86 \%$ of intervention versus $71 \%$ of control participants completing all visits. Intervention participants also had higher adherence (72\%) than control participants (57\%) based on TFV-DP levels indicating $\geq 4$ days per week dosing. The TAPIR and EPIC trials differed both by intervention type (automated versus interactive texting) and control patient adherence levels (high versus moderate), so differences in study outcomes may be due to either or both of these factors. These results merit further exploration, possibly in an implementation science framework to explore how to best scale up these promising interventions.

App and mobile-optimized web approaches are promising avenues for supporting PrEP initiation, although no clinical trial data are currently available regarding their efficacy. One pilot study, HealthMindr, developed an app with a diverse array of HIV prevention services: screening for PrEP and post-exposure prophylaxis; prevention recommendations; no-cost prevention mail kits for condoms, lubricant, and HIV self-tests; and prevention locator services [12]. During the four-month follow-up, a majority of study participants ordered condoms and HIV tests, and 9\% (8/86) initiated PrEP. A number of clinical trials that use mHealth interventions to promote PrEP initiation or retention in care are underway. One trial adapts HealthMindr to youth populations, using social cognitive theory and substantial formative work [13], and another based on the information, motivation, and behavioral skills' theory uses personalized risk scores, electronic diaries, and home testing to promote PrEP uptake [14]. A third trial focuses on retention in PrEP care by building on an app platform featuring social network and game-based components [15]. As results from these trials become available, it may be more clear which theoretical models and app services are best suited for supporting PrEP initiation and adherence.

\section{Alternate Dosing Strategies}

Daily PrEP dosing strategies are the only regimens with US Food and Drug Administration approval, but the World Health Organization, as well as health departments in New York City and San Francisco, have endorsed event-driven (ED) PrEP for MSM. This strategy has been covered in substantial depth elsewhere [16-21]. In brief, ED PrEP has most commonly been 4 pills of tenofovir disoproxil fumarate and emtricitabine (TDF/FTC): 2 pills $2-24 \mathrm{~h}$ before sex, 1 pill $24 \mathrm{~h}$ 


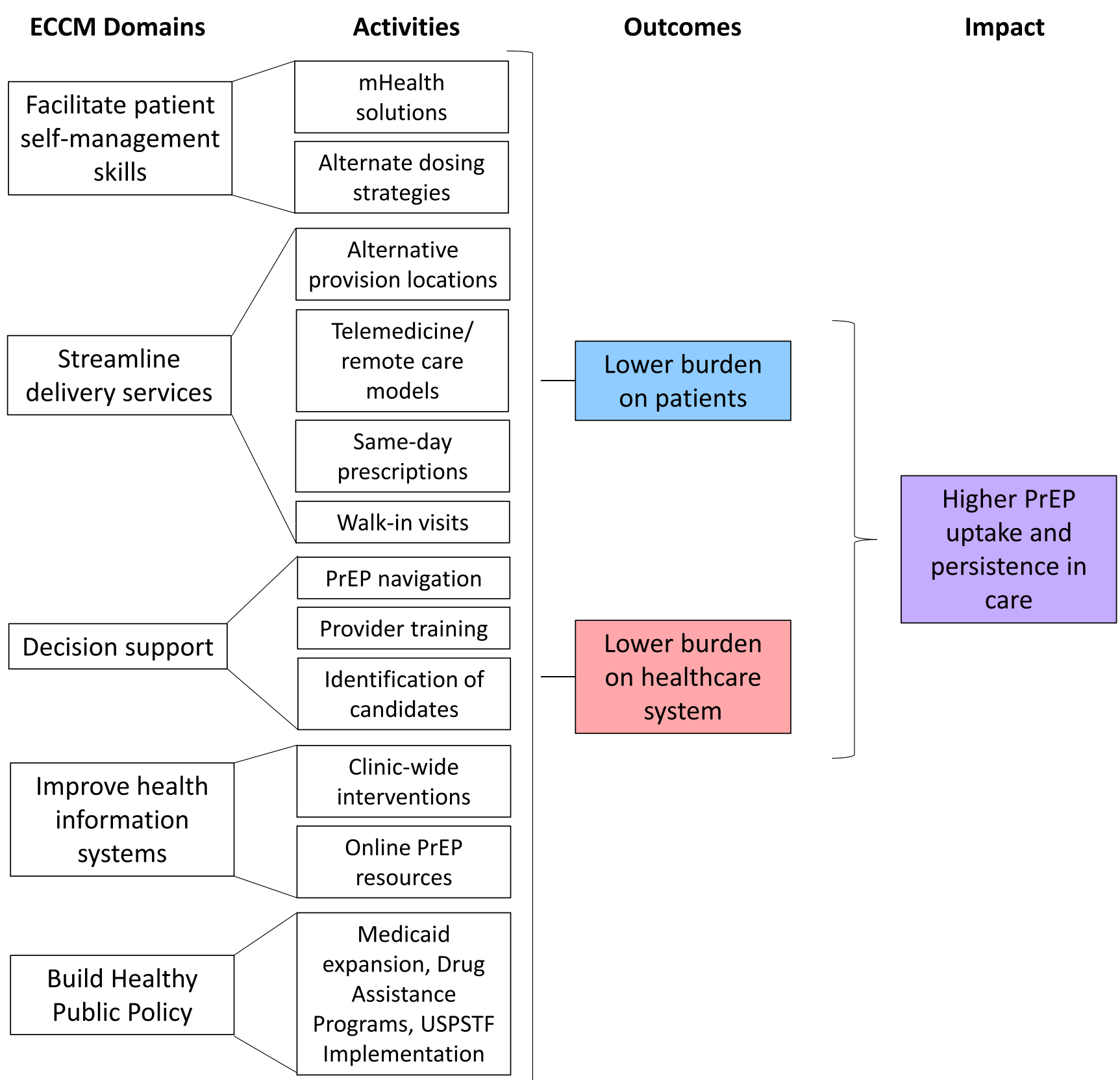

Fig. 1 Chronic care model applied to simplification of PrEP care

after the first dose, and 1 pill $48 \mathrm{~h}$ after the first dose (2-1-1). In the IPERGAY trial among MSM in France, as well as several subsequent open-label studies in Europe, ED PrEP performed exceptionally well, with an efficacy similar to daily PrEP [16-18]. ED PrEP performed less well in terms of adherence in HPTN067 among predominantly Black men at the Harlem site, with fewer sexual events covered with medication doses [22]. Modeling from these data indicates that scaling ED PrEP in lieu of daily PrEP might result in higher HIV transmission, particularly for minority MSM [23]. HPTN067 was conducted before the efficacy of event-driven PrEP was fully understood, though, which may limit the generalizability of these modeling results. Recently, no new HIV infections in 136 person years of follow-up were observed among a cohort of predominantly White and Asian ED PrEP users with Kaiser Permanente Insurance, with few reporting side effects or missed doses [24]. The eventual impact of ED PrEP scaleup, however, will depend on (1) the extent to which a clinician-recommended ED strategy could encourage PrEP use among persons who would otherwise not take it, (2) the proportion of daily users that might switch to a ED strategy, and (3) whether the ED strategy over the long-term would increase or decrease prevention-effective adherence as measured by coverage of HIV exposures. ED strategies have the potential to alleviate high rates of PrEP discontinuation among men; one national study in the USA found that only $56 \%$ of 
daily PrEP users persisted through 1 year of PrEP use and only $41 \%$ persisted through 2 years of use. Clinical studies have demonstrated that ED PrEP is efficacious when men are adherent to the dosing schedule, so it should receive substantial consideration as an option to facilitate population-level PrEP scale-up among MSM. It is important to note that this strategy has not yet been tested in women and that there are concerns that ED PrEP might be less efficacious in women or transgender persons receiving gender-affirming hormone therapy because of possible sex differences in the pharmacology of tenofovir-based PrEP.

\section{Streamline Delivery Services}

\section{Alternative Provision Locations}

PrEP is most often provided by clinicians in standard clinic settings. One concept to improve the patient experience is to prescribe PrEP from alternative sites. This has several potential advantages: (1) alternative sites may be more conveniently located compared with centralized clinics/hospitals, (2) some individuals may feel more comfortable seeking preventive care outside of a standard clinic visit, and (3) increased visibility of alternative sites may spur uptake of an otherwise unplanned behavior. These advantages are similar to those gained by HIV mobile testing units relative to clinic-based testing. Mobile HIV testing was found in one clinical trial to increase HIV testing by over 4 times [25] and has been observed to increase participation of important subpopulations such as first-time testers [26]. Encouragingly, PrEP provision through a mobile clinic such as a van is a feasible strategy for outreach to low-service areas; conducted among a predominantly Hispanic population, 168/225 mobile clinic clients sought PrEP and the majority sought a follow-up visit within 3 months [27]. Other promising venues are public health programs, community health centers, STI/HIV testing clinics, and other locations where at-risk persons receive care [28-30]. Each of these may offer unique opportunities to engage persons in considering PrEP care, which is especially important in light of the evidence that repeated offers of PrEP over time can facilitate PrEP initiation [31, 32].

Several studies have used collaborative practice agreements to allow pharmacists to prescribe PrEP from their pharmacies [33-35]. All studies found the programs to be highly acceptable and feasible, but among the two with follow-up data, one found high retention in care, [34] and the other found lower retention in care (28\% at 12 months) [33]. Also, mixed results were found for the ability to recruit a diverse patient population, with one having notable success in recruiting Latino MSM [35 ${ }^{\circ}$ and other studies not reporting or having predominantly White patient populations. Given legal regulations regarding collaborative practice agreements, such programs may not be scalable unless states change their laws regarding PrEP. Recently, California made just such a change, enacting California Senate Bill 159 that would allow pharmacists to prescribe to individuals a single, 60-day regimen of PrEP, which could lower the barrier of initiating PrEP [36].

Another model is for providers at acute or retail care clinics, often housed within pharmacies, to prescribe PrEP. Such clinics offer on-demand appointments that do not require scheduling or expectation of follow-up [37, 38]. Two pharmacy chains in the USA, Walgreens and CVS, have implemented PrEP care nationally in their retail care clinics, and the organizations have agreed to have their clinics listed in a national database of PrEP-prescribing clinics [39]. A literature review comparing the efficacy of retail care clinics vs. physician offices for chronic disease management revealed a lack of evidence-based data favoring either setting [37]. An additional literature review comparing retail care clinics with other sites of care in terms of cost, quality, and patient satisfaction found inconsistent measures across a total of 15 studies that greatly limited the ability to make definitive conclusions $[37,38]$. The reviews note the growing popularity of such clinics, yet indicate there is insufficient evidence to inform patients, clinicians, and policymakers in expanding the use of this important resource $[37,38]$.

\section{Telemedicine/Remote Care Models}

One step beyond alternative PrEP care sites is telemedicine, which could make in-person medical visits non-obligatory. There are a number of substantial potential benefits of remote care approaches for PrEP, including reduced transit time, effort, and cost; reduced potential stigma in seeking care; and lower burdens on busy clinical sites. Perhaps the most compelling is that having four in-person clinic visits each year specifically for using medications with an excellent safety profile could be unnecessary and unsustainable for many. Such burdens are not imposed for other well-tolerated medications such as oral contraceptives or statins, likely because it could adversely impact retention. When provided the hypothetical choice, the majority of participants in a national sample of MSM preferred home-based PrEP care to clinic-based care [40]. Two small pilot studies of telemedicine PrEP indicate feasibility and promise of the approach, although the small sample for each study limits larger conclusions [41, 42]. A randomized trial of telemedicine PrEP, using an appbased platform to facilitate the remote care, is currently underway and should provide more definitive evidence regarding the impact of telemedicine PrEP on initiation and maintenance in care [43]. To optimize the likelihood of impact, all aspects of PrEP care in the trial can be completed from home, including self-collection of specimens for laboratory testing and mailing of prescriptions when possible. A number of commercial telemedicine PrEP companies have emerged. For individuals who are underinsured, uninsured, or use Medicare/ 
Medicaid, payment barriers may exist to accessing such commercial services, although some note on their websites that payment assistance navigation is provided.

A different model of remote PrEP care seeks to reduce the number of required visits. For instance, a single annual inperson visit could be with a clinician, and three intervening visits could be performed by remote lab testing and an electronic survey. A pilot test of this model, conducted by the study authors, found that most participants preferred it to standard care and that $40 \%$ would be more willing to remain on PrEP if this option was provided [44]. A challenge for both full telemedicine PrEP and alternative reduced burden models is payment and billing. Addressing such issues could be accomplished with legislative or other systems-based changes and would facilitate implementation of telemedicine PrEP approaches.

\section{Same-Day Prescriptions}

Same-day prescription refers to PrEP initiation on the same day as a patient's first visit with a PrEP provider. Many clinicians currently use a referral model, with PrEP discussion and evaluation followed by either a referral to another clinic or a scheduled follow-up appointment for PrEP initiation after laboratory testing is complete. Referral models can introduce several days or more of delay between initial patient contact and PrEP initiation - with an inherent potential for loss to follow-up (i.e., primary non-adherence). For HIV treatment initiation, same-day prescriptions after positive testing have had success [45, 46]. Denver Public Health has constructed a model for same-day PrEP at walk-in STI clinics, including PrEP eligibility assessment, serum creatinine testing, hepatitis B testing, urine pregnancy testing, point-of-care HIV testing, patient navigator counseling, and 30-day PrEP prescription; when this system was used, $78 \%$ of patients returned for a 1month follow-up visit, and $57 \%$ returned for a follow-up visit at 3 months [47]. A New York City study that used medical chart review found that same-day initiation of PrEP was associated with decreased retention in care at the third follow-up visit [48]. The authors did not speculate on the reasons for this unexpected association. It is possible that more non-adherent patients were classified as same-day initiators relative to patients in the delay-care group (e.g., patients in the delay care group may have discussed PrEP with their clinician but never returned for a formal "PrEP initiation" visit, and therefore were counted as not starting rather than discontinuing). Thus, it is challenging to determine the overall impact of a same-day program without additional information to supplement a medical records' review. A study in Kenya developed an approach for same-day PrEP among pregnant and postpartum women, with assessment ongoing [49]. Additional studies are needed to determine the benefits and potential limitations of same-day PrEP programs.

\section{Walk-In Visits}

Scheduling of a healthcare visit for persons who are frequently healthy and young, and therefore potentially unaccustomed to scheduling regular clinician visits, may be a barrier to getting PrEP. Many STI and HIV testing sites already exist as walk-in clinics to address this challenge. These anonymous clinics do not require scheduled visits during regular hours, and these sites are often preferred among key populations such as men who have sex with men (MSM) and persons who engage in sex work [50]. A survey at the Harris Health System in Houston Texas found that individuals $\geq 18$ years old presenting for walk-in HIV testing expressed interest in taking PrEP at a rate of about $73 \%$ [51], suggesting an opportunity to engage patients in PrEP after walk-in HIV testing. PrEP studies conducted by Denver Public Health utilized walk-in visits for PrEP initiation [47]; however, no comparisons exist with appointment-based models. Moreover, subsequent PrEP care follow-up visits are scheduled months in advance, potentially easing the challenge of visit scheduling. Further assessments of walk-in visit models for PrEP care are needed, given demonstrated interest among key populations and limited data for its application to sexual health.

\section{Decision Support}

\section{PrEP Navigation}

In general, patient navigation is defined as service provided by non-clinical staff members to assist patients in addressing barriers to PrEP care [52]. PrEP navigation initiatives are often comprised of trusted peers who possess shared lived experiences with the community and are frequently adopted and supported by clinics with large numbers of PrEP patients. [52, 53] These services can also be used to offset time burden on clinicians, thereby alleviating a potential barrier to clinician capacity and willingness to prescribing PrEP. Several studies explored PrEP navigation in implementation or pilot study settings, indicating success for various types of PrEP navigation with obtaining higher PrEP adherence or earlier PrEP initiation [54-56]. Studies of PrEP navigation and its utility are ongoing, with a review by Pinto et al. noting at least $6 \mathrm{NIH}-$ funded studies of "PrEP navigation" focusing on "black MSM, young Latino MSM, women upon release from incarceration, people who inject drugs (PWID), and methamphetamine users." [53] A number of online resources for PrEP navigation may facilitate its adoption into the practice of providers currently prescribing PrEP. For instance, PleasePrEPMe and the NYC Health Department offer training for various methods of PrEP navigation, featuring online courses and detailed manuals of operation [57-59]. 


\section{Provider Training}

Despite the Centers for Disease Control and Prevention (CDC) first publishing draft PrEP practice guidelines in 2012 and releasing formal clinical guidelines in 2014, a recent survey of primary care providers (PCPs) identified that although most PCPs were interested in prescribing PrEP, only $17 \%$ of PCPs had ever prescribed it, and only $33 \%$ had ever discussed it with patients $[60,61]$. Reasons identified for low levels of PrEP discussion and prescription included limited knowledge of PrEP and concerns about insurance and other barriers. An additional barrier to PrEP provision by PCPs has been described as a "purview paradox," in which PCPs generally view PrEP prescription as a responsibility of HIV specialists-whereas most HIV negative patients who are eligible for PrEP do not possess any need for such specialized care [62]. Low PrEP prescribing is not unique to PCPs; a national survey of family planning providers, a group with specialized expertise in sexual health services, found low PrEP knowledge and use overall among this group [63]. Only one-third of respondents could correctly define PrEP and its efficacy, and less than 5\% had ever prescribed PrEP. The majority felt uncomfortable prescribing PrEP due to lack of training.

A literature review of provider educational interventions for increasing PrEP implementation in primary care settings lists suggestions of clinical scenarios that should be included for provider training [64], and qualitative studies provide useful information regarding which modalities for training are preferred among providers [65]. A single 20-60-min inperson educational presentation at 14 Duke PCP offices resulted in a fourfold increase (OR 4.8) in self-reported PrEP prescriptions among physicians who attended the session [66]. Similarly, a 1.5-h in-person educational training at 4 family planning clinics in Metro Atlanta resulted in increased provider PrEP knowledge and confidence to identify women who may benefit from PrEP [67]. Post-training exit interviews with patients revealed that providers discussed HIV risk and PrEP with the majority of women with PrEP indications. Encouragingly, participants in a PrEP-based extension of the Project ECHO (Extension for Community Healthcare Outcomes) telementoring program had improved knowledge and reported increased likelihood of prescribing PrEP two years after their training [68]. Other in-depth PrEP provider training support resources exist, such as an online, continuing medical education course ("PrEParing") created at Johns Hopkins University that exposes learners to $12 \mathrm{~h}$ of content over 6 weeks [69]. For more complex medical issues or questions, the University of California, San Francisco, offers a support line for clinicians with questions about PrEP [70]. Although the most effective timing, content, and modality of PrEP provider education are not yet clear, it is likely that a variety of options may be beneficial to meet individual preferences and knowledge deficits.

\section{Identification of PrEP Candidates}

Ideally, clinical providers would elicit comprehensive sexual and substance use histories with all of their patients as part of routine healthcare, which would facilitate identification of persons who are likely to benefit from PrEP use. Given competing priorities and intensive time constraints, though, providers do not typically ascertain indications for PrEP for many of their patients.

Several tools have been developed that could help streamline the process by which providers identify candidates for PrEP. Clinical prediction rules have been developed and validated to identify persons at increased risk for future HIV acquisition based on self-reported demographic and behavioral questions, such as age, race, and sexual and substance use behaviors. These tools could be used at the point-of-care to identify people for discussions about PrEP. However, these tools have only been developed for MSM and people who inject drugs (i.e., not for transgender people or cisgender women), they have suboptimal predictive accuracy [71-74], and some have lower sensitivity for specific populations (e.g. black MSM) [75, 76], all of which could limit their utility.

An additional strategy for identifying PrEP candidates is to use routine electronic health records (EHR) data to alert providers about individual patients at increased risk for HIV acquisition. These methods, unlike the prediction tools mentioned above, have the benefit of using existing components of health records rather than screening data. This avoids having clinicians potentially serving as PrEP gate-keepers, potentially democratizing the selection process if implemented appropriately. Studies have used machine learning methodologies to identify individuals at increased risk for incident HIV in large healthcare systems using EHR data [77, 78]. These automated prediction algorithms have had high predictive performance for identifying men who may benefit from PrEP use in general care settings, with predictive accuracy that is comparable with that of prediction rules commonly used in other areas of preventive medicine (e.g., the Framingham Risk Score) [79]. However, prediction models have had poor performance at identifying women in these settings thus far, which could be in part because the models were trained using data that included very few women with incident HIV. Additional studies to test the impact of implementing EHR prediction models on PrEP use and to improve their performance for women are underway. Moreover, these tools must be implemented carefully and, in awareness of existing disparities by race/ethnicity, ideally explicitly targeted to minimize care access disparities. 


\section{Improve Health Information Systems}

\section{Clinic-Wide Interventions}

Slow adoption of any new evidence-based practice, like PrEP, into clinical care is a widespread concern among healthcare systems [80, 81], with some noting an average 15 - to 20 -year lag before a new evidence-based practice is widely integrated into routine care [82]. Clinics have difficulty systematically implementing new practices, and often this is due to challenges coordinating change across multiple aspects of a practice setting, rather than their lack of recognizing a new practice as relevant and desirable to provide $[80,83]$. PrEP care requires a higher level of engagement of healthcare providers, along with clinic support staff, than has typically been required for HIV prevention (e.g., condom-focused), and has cost and resource considerations relevant for clinics. The steps in PrEP delivery include the following: (1) eliciting a patient's sexual and substance use history to inform the potential benefits of PrEP use, (2) providing PrEP counseling, (3) assessing a patient's candidacy for PrEP via labs, (4) prescribing PrEP, and (5) follow-up and clinical monitoring. During Step 1, the patients are screened for HIV risk factors and tested for HIV (noting that HIV testing could also happen during Step 3). During Step 2, the patients are provided with information about PrEP and their interest and potential to adhere to PrEP are assessed. During Step 3, the patients are assessed for any signs and symptoms of undiagnosed HIV infection and receive laboratory testing for kidney function, hepatitis B and C, pregnancy (when relevant), and other STIs. Step 4 involves prescribing PrEP to patients who meet clinical criteria and express interest. This step also may involve enrolling the patient in insurance or medication assistance programs to ensure they can pay for PrEP, which can involve intensive health navigation for some patients. Step 5 involves follow-up visits every 3 months for HIV testing, adherence counseling, risk reduction support, side effect assessment, pregnancy testing, STI testing, and kidney function testing. Due to differences in organizational structure, services provided, community partnerships, capacity (staffing and financial), and resources, clinics may require varying levels of intervention focused on one or more of the aforementioned steps to support the delivery of onsite PrEP care [84].

Clinic-level interventions consist of varying implementation strategies to enhance the adoption, implementation, and sustainability of a new clinical practice. Powell et al. have characterized implementation strategies into 6 groups: planning (e.g., conducting a clinic-level organizational assessment, developing a formal implementation plan), educating (e.g., conduct educational trainings, distribute educational materials), financing (e.g., access new funding), restructuring (e.g., task-shifting), managing quality (e.g., provide clinical supervision, audit and feedback, EHR or patient reminder systems), and attending to policy context (e.g., creating or changing scope of practice restrictions) [85]. Often a combination of implementation strategies is required to achieve clinic-wide practice change [86]. The majority of studies among providers have concluded that more education about PrEP and its delivery and access to tools (e.g., HIV risk assessment screeners, EHR-based risk algorithms, patient educational resources) are needed to optimize PrEP implementation [62, 87-89]. Even as knowledge about PrEP increases, prescribing providers may still have concerns about how adding PrEP counseling, ordering/reviewing labs, and monitoring would add substantial time to limited visit allowance per patient. Task-shifting models of PrEP care, such as those employing patient navigators to support patient education and financial navigation, have been successfully implemented, even in resource-constrained environments like safety-net community clinics [53-56]. Robust evaluations of PrEP implementation plans that distribute the tasks associated with PrEP (e.g., screening, education, testing, and monitoring) across various clinic staff to offset the burden of PrEP care falling on providers are warranted. Given the multiple steps of PrEP care, it is likely that more than one implementation strategy will be necessary to facilitate scaling PrEP in various clinical or alternative care environments. Encouragingly, innovative combination interventions, like Saberi and colleagues' program consisting of patient support staff to handle screening and insurance navigation in addition to a web-based management tool to facilitated patient follow-up, have recently been developed [54]. However, there is a need for rigorous evaluations of PrEP implementation interventions, particularly those that combine multiple implementation strategies.

\section{Online PrEP Resources}

As clinics and the health systems make changes to enhance access to PrEP care, it is important for online resources to similarly facilitate access. One such resource is the community-based Facebook group PrEP Facts [90], which has over 20,000 members and seeks to support persons interested in or on PrEP. The moderated group, founded by Damon L. Jacobs, has participation via request in order to increase privacy and facilitate sharing of accurate information. Another resource is PrEP Locator, a national listing of PrEP providers in the USA that was created by the authors and is now supported by the US Centers for Disease Control and Prevention [39]. The PrEP Locator website has had over one million views and over one-half million users since its launch in late 2016, in spite of no advertising budget for the site. A pilot user study found that most users $(48 / 54,89 \%)$ expected the site to be useful in the future, over one-third reported that the site helped them find a provider (21/54), and over $10 \%$ (7/54) had moved forward on the PrEP care cascade by either scheduling or completing a PrEP initiation visit within 
1 month of visiting the site [91]. The use and utility of this site indicate the promise of novel and easily accessible online resources. Another freely available online resource, the "What is PrEP" video, which provides a detailed and accessible description of PrEP and PrEP care, has been viewed over 200,000 times according to its website tracker. Given the high interest in and use of these free online resources, future interventions that seek to leverage them to enhance engagement in the PrEP care cascade represent a promising choice worthy of implementation science research.

\section{Building Healthy Public Policy}

Laws and their implementation intersect with PrEP care and services in concrete ways that can facilitate or impede PrEP use [92]. At the local level, some health departments make PrEP referrals, provide education to the public or medical providers, or even prescribe PrEP directly through health department clinics [93]. At the state level, PrEP Drug Assistance Programs (PrEP-DAP) are offered by a number of states and cover medication costs or ancillary costs such as laboratory testing and clinical visit fees. Separately, Medicaid expansion policies have led to increased numbers of persons accessing health insurance coverage [94]. One recent study in the USA found that the states that had either PrEP-DAP or expanded Medicaid had 25\% higher PrEP prescriptions per population and that the states that had enacted both policies had $99 \%$ higher PrEP prescriptions per population [95]. At the national level, the US Preventive Services Task Force in 2019 rated PrEP with their highest recommendation (Grade A), meaning that there is "high certainty that the net benefit is substantial." [96] This rating has considerable implications, because it requires that commercial insurers cover the intervention medication cost without any cost sharing. It is currently unclear whether this coverage would also include the ancillary services that are required to receive a PrEP prescription [97].

\section{Conclusion}

Streamlining PrEP care has the potential to improve initiation of and persistence on PrEP, which are the major barriers to PrEP having an optimal population-level impact. The ECCM provided a useful framework to consider the panoply of interventions that could contribute to optimizing PrEP delivery. This review identified a number of promising strategies, including evidence-based interventions. There is a need to further develop an evidence base regarding other PrEP streamlining modalities, with future evidence to be created through future clinical trials or rigorously evaluated implementation science studies. These efforts should include costeffectiveness assessments to inform future resource allocation strategies. In the meantime, programs should seek to streamline their procedures in accordance with the needs of their clients. Many clinics are currently offering same-day PrEP, using dedicated PrEP navigators, or reducing visit schedules. The laudable goal of these programs is to provide patient-centered care that reduces barriers to PrEP, and we urge that these efforts continue as an evidence base is built to inform which strategies are most efficacious.

Funding This research was supported in part by the National Institute of Mental Health (R01MH114692) and by the National Institute of Allergy and Infectious Diseases (R01AI143875). The work was facilitated by the Emory Center for AIDS Research (P30AI050409).

\section{Compliance with Ethical Standards}

Conflict of Interest Aaron Siegler is a co-investigator on a grant from Gilead Foundation, paid to his institution. Kevin Steehler declares no conflict of interest. Jessica Sales received a grant from Gilead Foundation in the past 5 years, paid to her institution. Douglas Krakower has conducted research with project support from Gilead Sciences; has received honoraria for authoring or presenting continuing medical education content for Medscape, MED-IQ, and DKBMed; and has received royalties for authoring content for UpToDate, Inc.

Human and Animal Rights and Informed Consent This article does not contain any studies with human or animal subjects performed by any of the authors.

Open Access This article is licensed under a Creative Commons Attribution 4.0 International License, which permits use, sharing, adaptation, distribution and reproduction in any medium or format, as long as you give appropriate credit to the original author(s) and the source, provide a link to the Creative Commons licence, and indicate if changes were made. The images or other third party material in this article are included in the article's Creative Commons licence, unless indicated otherwise in a credit line to the material. If material is not included in the article's Creative Commons licence and your intended use is not permitted by statutory regulation or exceeds the permitted use, you will need to obtain permission directly from the copyright holder. To view a copy of this licence, visit http://creativecommons.org/licenses/by/4.0/.

\section{References}

Papers of particular interest, published recently, have been highlighted as:

- Of importance

-• Of major importance

1. Grant RM, Lama JR, Anderson PL, McMahan V, Liu AY, Vargas L, et al. Preexposure chemoprophylaxis for HIV prevention in men who have sex with men. N Engl J Med. 2010;363(27):2587-99.

2. Fitch L, Clancy J, Donaldson E, Gardiner E, Warren M. Tracking global oral PrEP provision: the who, what, where of oral PrEP. Paper presented at: HIVR4P: HIV Research for Prevention conference October, 2018; Madrid.

3. Scheer S, Hsu L, Schwarcz S, et al. Trends in the San Francisco human immunodeficiency virus epidemic in the "getting to zero" era. Clin Infect Dis. 2017;66(7):1027-34. 
4. Centers for Disease Control and Prevention. US Public Health Service: preexposure prophylaxis for the prevention of HIV infection in the United States - 2017 update: a clinical practice guideline. 2018

5. World Health Organization. Consolidated guidelines on the use of antiretroviral drugs for treating and preventing HIV infection: recommendations for a public health approach. WHO Web site. http:// www.who.int/iris/handle/10665/208825. Published 2016. Accessed.

6. Barr V, Robinson S, Marin-Link B, Underhill L, Dotts A, Ravensdale D, et al. The expanded chronic care model: an integration of concepts and strategies from population health promotion and the chronic care model. Hospital Q. 2003;7:73-82.

7. Coleman K, Austin BT, Brach C, Wagner EH. Evidence on the chronic care model in the new millennium. Health Aff (Millwood). 2009;28(1):75-85.

8. Wagner EH, Austin BT, Von Korff M. Organizing care for patients with chronic illness. Milbank Q. 1996;74(4):511-44.

9. Horvath T, Azman H, Kennedy GE, Rutherford GW. Mobile phone text messaging for promoting adherence to antiretroviral therapy in patients with HIV infection. Cochrane Database Syst Rev (Online). 2012(3):Cd009756.

10. Moore DJ, Jain S, Dube MP, et al. Randomized controlled trial of daily text messages to support adherence to preexposure prophylaxis in individuals at risk for human immunodeficiency virus: the TAPIR study. Clin Infect Dis : an official publication of the Infectious Diseases Society of America. 2018;66(10):1566-72.

11.• Liu AY, Vittinghoff E, von Felten P, et al. Randomized controlled trial of a mobile health intervention to promote retention and adherence to preexposure prophylaxis among young people at risk for human immunodeficiency virus: the EPIC study. Clin Infect Dis : an official publication of the Infectious Diseases Society of America. 2019;68(12):2010-7 This clinical trial demonstrated that an interactive text message platfrom led to an increase in effective PrEP use ( $\geq 4$ days per week).

12. Sullivan PS, Driggers R, Stekler JD, Siegler A, Goldenberg T, McDougal SJ, et al. Usability and acceptability of a mobile comprehensive HIV prevention app for men who have sex with men: a pilot study. JMIR Mhealth Uhealth. 2017;5(3):e26.

13. Biello KB, Marrow E, Mimiaga MJ, Sullivan P, HightowWeidman L, Mayer KH. A mobile-based app (MyChoices) to increase uptake of HIV testing and pre-exposure prophylaxis by young men who have sex with men: protocol for a pilot randomized controlled trial. JMIR Res Protocol. 2019;8(1):-e10694.

14. Liu A, Coleman K, Bojan K, Serrano PA, Oyedele T, Garcia A, et al. Developing a mobile app (LYNX) to support linkage to HIV/ sexually transmitted infection testing and pre-exposure prophylaxis for young men who have sex with men: protocol for a randomized controlled trial. JMIR Res Protoc. 2019;8(1):e10659.

15. LeGrand S, Knudtson K, Benkeser D, Muessig K, Mcgee A, Sullivan PS, et al. Testing the efficacy of a social networking gamification app to improve pre-exposure prophylaxis adherence (P3: prepared, protected, emPowered): protocol for a randomized controlled trial. JMIR Res Protoc. 2018;7(12):e10448.

16. Molina JM, Capitant C, Spire B, Pialoux G, Cotte L, Charreau I, et al. On-demand preexposure prophylaxis in men at high risk for HIV-1 infection. N Engl J Med. 2015;373(23):2237-46.

17. Molina J-M, Ghosn J, Algarte-Genin M, et al. Incidence of HIVinfection with daily or on-demand PrEP with TDF/FTC in Paris area. Update from the ANRS Prevenir Study. Paper presented at: J Int Aids Soc 2019.

18. Hoornenborg E, Coyer L, Achterbergh RC, Matser A, Schim van der Loeff M, Boyd A, et al. Sexual behaviour and incidence of HIV and sexually transmitted infections among men who have sex with men using daily and event-driven pre-exposure prophylaxis in
AMPrEP: 2 year results from a demonstration study. The Lancet HIV. 2019;6:e447-55.

19. Reyniers T, Nöstlinger C, Laga M, de Baetselier I, Crucitti T, Wouters $\mathrm{K}$, et al. Choosing between daily and event-driven preexposure prophylaxis: results of a Belgian PrEP demonstration project. JAIDS J Acquir Immune Defic Syndr. 2018;79(2):186-94.

20. Cohen SE, Kelley CF. Is the United States ready for event-driven human immunodeficiency virus Preexposure prophylaxis? Clin Infect Diseases. 2019.

21. Bauer R, Netzer E, Pintado C, et al. Coverage of sex acts by eventdriven pre-exposure prophylaxis: a sub-study of the ANRS IPERGAY trial. AIDS Behav 2020.

22. Grant RM, Mannheimer S, Hughes JP, Hirsch-Moverman Y, Loquere A, Chitwarakorn A, et al. Daily and nondaily oral preexposure prophylaxis in men and transgender women who have sex with men: the human immunodeficiency virus prevention trials network 067/ADAPT study. Clin Infect Dis : an official publication of the Infectious Diseases Society of America. 2018;66(11):171221.

23. Dimitrov D, Moore JR, Wood D, et al. Predicted effectiveness of daily and nondaily preexposure prophylaxis for men who have sex with men based on sex and pill-taking patterns from the human immuno virus prevention trials network 067/ADAPT study. Clin Infect Dis 2019.

24. Hojilla JC, Marcus JL, Silverberg MJ, et al. Early adopters of eventdriven human immunodeficiency virus pre-exposure prophylaxis in a large healthcare system in San Francisco. Clin Infect Diseases. 2020.

25. Khumalo-Sakutukwa G, Morin SF, Fritz K, et al. Project Accept (HPTN 043): a community-based intervention to reduce HIV incidence in populations at risk for HIV in sub-Saharan Africa and Thailand. J Acquir Immune Defic Syndr (1999). 2008;49(4):42231.

26. Lipsitz MC, Segura ER, Castro JL, Smith E, Medrano C, Clark JL, et al. Bringing testing to the people - benefits of mobile unit HIV/ syphilis testing in Lima, Peru, 2007-2009. Int J STD AIDS. 2014;25(5):325-31.

27. Doblecki-Lewis S, Kobetz E. Byrne, et al. 1965. PrEP on the go! Implementation Mobile PrEP, STI, and HIV prevention services in South Florida. Open Forum Infect Diseases. 2019;6(Suppl 2):S65.

28. Liu A, Cohen S, Follansbee S, Cohan D, Weber S, Sachdev D, et al. Early experiences implementing pre-exposure prophylaxis (PrEP) for HIV prevention in San Francisco. PLoS Med. 2014;11(3): e1001613.

29. Kamis KF, Marx GE, Scott KA, et al. Same-day HIV pre-exposure prophylaxis (PrEP) initiation during drop-in sexually transmitted diseases clinic appointments is a highly acceptable, feasible, and safe model that engages individuals at risk for HIV into PrEP care. Open Forum Infect Diseases. 2019;6(7).

30. Mayer KH, Chan PA. R RP, Flash CA, Krakower DS. Evolving models and ongoing challenges for HIV preexposure prophylaxis implementation in the United States. J Acquir Immune Defic Syndr. 2018;77(2):119-27.

31. Lockard A, Rosenberg ES, Sullivan PS, Kelley CF, Serota DP, Rolle CPM, et al. Contrasting self-perceived need and guidelinebased indication for HIV pre-exposure prophylaxis among young, black men who have sex with men offered pre-exposure prophylaxis in Atlanta, Georgia. AIDS Patient Care STDs. 2019;33(3): $112-9$.

32. Serota DP, Rosenberg ES, Sullivan PS, et al. Pre-exposure prophylaxis uptake and discontinuation among young black men who have sex with men in Atlanta, Georgia: a prospective cohort study. Clin Infect Diseases : an official publication of the Infectious Diseases Society of America. 2019.

33. Havens JP, Scarsi KK, Sayles H, Klepser DG, Swindells S, Bares $\mathrm{SH}$. Acceptability and feasibility of a pharmacist-led HIV pre- 
exposure prophylaxis (PrEP) program in the Midwestern United States. Open Forum Infect Dis. 2019;6(10).

34. Tung EL, Thomas A, Eichner A, Shalit P. Implementation of a community pharmacy-based pre-exposure prophylaxis service: a novel model for pre-exposure prophylaxis care. Sex Health. 2018;15(6):556-61.

35. Lopez MI, Cocohoba J, Cohen SE, Trainor N, Levy MM, Dong BJ. Implementation of pre-exposure prophylaxis at a community pharmacy through a collaborative practice agreement with San Francisco Department of Public Health. J Am Pharm Assoc (2003). 2019;60(1):138-44 This study demonstrates the abiltiy of a pharmacy-based PrEP provision program to initiate a diverse group of MSM into PrEP care.

36. SB-159 HIV: preexposure and postexposure prophylaxis.(20192020). In: California, ed 2019.

37. Chen CE, Chen $\mathrm{CT}, \mathrm{Hu}$ J, Mehrotra A. Walk-in clinics versus physician offices and emergency rooms for urgent care and chronic disease management. Cochrane Database Syst Rev. 2017;2: CD011774.

38. Hoff T, Prout K. Comparing retail clinics with other sites of care: a systematic review of cost, quality, and patient satisfaction. Med Care. 2019;57(9):734-41.

39. Siegler AJ, Wirtz S, Weber S, Sullivan PS. Developing a web-based geolocated directory of HIV pre-exposure prophylaxis-providing clinics: the PrEP locator protocol and operating procedures. JMIR Public Health Surveill. 2017;3(3):e58.

40. John SA, Rendina HJ, Grov C, Parsons JT. Home-based pre-exposure prophylaxis (PrEP) services for gay and bisexual men: an opportunity to address barriers to PrEP uptake and persistence. PLoS One. 2017;12(12):e0189794.

41. Siegler AJ, Brock JB, Kelley CF, et al. Pilot test of a PrEP telemedicine system for young, black MSM in the rural US south. Seattle: CROI; 2019.

42. Refugio ON, Kimble MM, Silva CL, Lykens JE, Bannister C, Klausner JD. Brief report: PrEPTECH: a telehealth-based initiation program for HIV pre-exposure prophylaxis in young men of color who have sex with men. A pilot study of feasibility. JAIDS J Acquir Immune Defic Syndr. 2019;80(1):40-5.

43. Siegler AJ, Brock JB, Hurt CB, Ahlschlager L, Dominguez K, Kelley CF, et al. An electronic pre-exposure prophylaxis initiation and maintenance home care system for nonurban young men who have sex with men: protocol for a randomized controlled trial. JMIR Res Protoc. 2019;8(6):e13982.

44. Siegler AJ, Mayer KH, Liu AY, Patel RR, Ahlschlager LM, Kraft CS, et al. Developing and assessing the feasibility of a home-based PrEP monitoring and support program. Clin Infect Diseases : an official publication of the Infectious Diseases Society of America. 2019;68(3):501-4

45. Koenig SP, Dorvil N, Devieux JG, et al. Same-day HIV testing with initiation of antiretroviral therapy versus standard care for persons living with HIV: a randomized unblinded trial. PLoS Med. 2017;14(7):e1002357.

46. Pilcher CD, Ospina-Norvell C, Dasgupta A, Jones D, Hartogensis $\mathrm{W}$, Torres S, et al. The effect of same-day observed initiation of antiretroviral therapy on HIV viral load and treatment outcomes in a US public health setting. J Acquir Immune Defic Syndr. 2017;74(1):44-51.

47. Kamis KF, Marx GE, Scott KA, et al. Same-day HIV pre-exposure prophylaxis (PrEP) initiation during drop-in sexually transmitted diseases clinic appointments is a highly acceptable, feasible, and safe model that engages individuals at risk for HIV into PrEP care. Open Forum Infect Dis. 2019;6(7):ofz310.

48. Zucker J, Carnevale C, Richards P, Slowikowski J, Borsa A, Gottlieb F, et al. Predictors of disengagement in care for individuals receiving pre-exposure prophylaxis (PrEP). J Acquir Immune Defic Syndr. 2019;81(4):e104-8.
49. Pintye J, Kinuthia J, Roberts DA, Wagner AD, Mugwanya K, Abuna F, et al. Brief report: integration of PrEP services into routine antenatal and postnatal care: experiences from an implementation program in Western Kenya. J Acquir Immune Defic Syndr. 2018;79(5):590-5.

50. Underhill K, Morrow KM, Colleran CM, Holcomb R, Operario D, Calabrese SK, et al. Access to healthcare, HIV/STI testing, and preferred pre-exposure prophylaxis providers among men who have sex with men and men who engage in street-based sex work in the US. PLoS One. 2014;9(11):e112425.

51. Flash CA, Adegboyega OO, Yu X, Avalos C, Johnson S, Mayer $\mathrm{KH}$, et al. Correlates of linkage to HIV preexposure prophylaxis among HIV-testing clients. J Acquir Immune Defic Syndr. 2018;77(4):365-72.

52. Mugavero MJ, Amico KR, Horn T, Thompson MA. The state of engagement in HIV care in the United States: from cascade to continuum to control. Clin Infect Diseases : an official publication of the Infectious Diseases Society of America. 2013;57(8):1164 71.

53. Pinto RM, Berringer KR, Melendez R, Mmeje O. Improving PrEP implementation through multilevel interventions: a synthesis of the literature. AIDS Behav. 2018;22(11):3681-91.

54. Saberi P, Berrean B, Thomas S, Gandhi M, Scott H. A simple preexposure prophylaxis (PrEP) optimization intervention for health care providers prescribing PrEP: pilot study. JMIR Form Res. 2018;2(1):e2.

55. Spinelli MA, Scott HM, Vittinghoff E, Liu AY, Morehead-Gee A, Gonzalez R, et al. Brief report: a panel management and patient navigation intervention is associated with earlier PrEP initiation in a safety-net primary care health system. J Acquir Immune Defic Syndr. 2018;79(3):347-51.

56. Reback CJ, Clark KA, Runger D, Fehrenbacher AE. A promising PrEP navigation intervention for transgender women and men who have sex with men experiencing multiple syndemic health disparities. J Community Health. 2019;44(6):1193-203.

57. PleasePrEPMe. A frontline provider training on PrEP research, care, and navigation. https://www.pleaseprepme.org/ PrEPNavTraining. Published 2019. Accessed December 12, 2019.

58. Hubbard S Scaling Up PrEP. NYC Department of Health. https:// www1.nyc.gov/assets/doh/downloads/pdf/dires/scaling-up-prepin-nyc.pdf. Published 2019. Accessed December 13, 2019.

59. PleasePrEPMe. Helping people access pre-exposure prophylaxis, a frontline provider manual on PrEP research, care, and navigation. https://www.avac.org/sites/default/files/resource-files/PrEP Navigator_Manual.pdf. Published 2019. Accessed December 13, 2019.

60. CDC. US Public Health Service: preexposure prophylaxis for the prevention of HIV infection in the United States - 2017 update: a clinical practice guideline. https://www.cdc.gov/hiv/pdf/risk/prep/ cdc-hiv-prep-guidelines-2017.pdf. Published 2017. Accessed December 13, 2019.

61. Petroll AE, Walsh JL, Owczarzak JL, McAuliffe TL, Bogart LM, Kelly JA. PrEP awareness, familiarity, comfort, and prescribing experience among US primary care providers and HIV specialists. AIDS Behav. 2017;21(5):1256-67.

62. Krakower D, Ware N, Mitty JA, Maloney K, Mayer KH. HIV providers' perceived barriers and facilitators to implementing preexposure prophylaxis in care settings: a qualitative study. AIDS Behav. 2014;18(9):1712-21.

63. Seidman D, Carlson K, Weber S, Witt J, Kelly PJ. United States family planning providers' knowledge of and attitudes towards preexposure prophylaxis for HIV prevention: a national survey. Contraception. 2016;93(5):463-9.

64. Silapaswan A, Krakower D, Mayer KH. Pre-exposure prophylaxis: a narrative review of provider behavior and interventions to 
increase PrEP implementation in primary care. J Gen Intern Med. 2017;32(2):192-8.

65. Calabrese SK, Magnus M, Mayer KH, et al. Putting PrEP into practice: lessons learned from early-adopting U.S. providers' firsthand experiences providing HIV pre-exposure prophylaxis and associated care. PLoS One. 2016;11(6): 0157324.

66. Clement ME, Seidelman J, Wu J, Alexis K, McGee K, Okeke NL, et al. An educational initiative in response to identified PrEP prescribing needs among PCPs in the Southern U.S. AIDS Care. 2018;30(5):650-5.

67. Sales JM, Cwiak C, Haddad LB, Phillips A, Powell L, Tamler I, et al. Brief report: impact of PrEP training for family planning providers on HIV prevention counseling and patient interest in PrEP in Atlanta, Georgia. J Acquir Immune Defic Syndr. 2019;81(4):414-8.

68. Wood BR, Mann MS, Martinez-Paz N, Unruh KT, Annese M, Spach DH, et al. Project ECHO: telementoring to educate and support prescribing of HIV pre-exposure prophylaxis by community medical providers. Sex Health. 2018;15(6):601-5.

69. University JH. PrEParing: PrEP for providers and patients. http:// www.coursera.org/learn/prep. Published 2019. Accessed December 13, 2019.

70. University of California San Francisco: Clinician Consultation Center. Introducing the CCC PrEPLine. https://nccc.ucsf.edu/ 2014/09/29/introducing-the-ccc-prepline/. Published 2014. Accessed Dec 23, 2019.

71. Scott H, Vittinghoff E, Irvin R, Liu A, Nelson LR, del Rio C, et al. Development and validation of the personalized sexual health promotion (SexPro) HIV risk prediction model for men who have sex with men in the United States. AIDS Behav. 2020;24(1):274-83.

72. Wilton J, Kain T, Fowler S, Hart TA, Grennan T, Maxwell J, et al. Use of an HIV-risk screening tool to identify optimal candidates for PrEP scale-up among men who have sex with men in Toronto, Canada: disconnect between objective and subjective HIV risk. J Int AIDS Soc. 2016;19(1):20777.

73. Smith DK, Pan Y, Rose CE, Pals SL, Mehta SH, Kirk GD, et al. A brief screening tool to assess the risk of contracting HIV infection among active injection drug users. J Addict Med. 2015;9(3):22632.

74. Smith DK, Pals SL, Herbst JH, Shinde S, Carey JW. Development of a clinical screening index predictive of incident HIV infection among men who have sex with men in the United States. J Acquir Immune Defic Syndr. 2012;60(4):421-7.

75. Lancki N, Almirol E, Alon L, McNulty M, Schneider JA. Preexposure prophylaxis guidelines have low sensitivity for identifying seroconverters in a sample of young Black MSM in Chicago. Aids. 2018;32(3):383-92.

76. Jones J, Hoenigl M, Siegler AJ, Sullivan PS, Little S, Rosenberg E. Assessing the performance of 3 human immunodeficiency virus incidence risk scores in a cohort of Black and White men who have sex with men in the south. Sex Transm Dis. 2017;44(5):297-302.

77. Marcus JL, Hurley LB, Krakower DS, Alexeeff S, Silverberg MJ, Volk JE. Use of electronic health record data and machine learning to identify candidates for HIV pre-exposure prophylaxis: a modelling study. The lancet HIV. 2019;6(10):e688-95.

78. Krakower DS, Gruber S, Hsu K, Menchaca JT, Maro JC, Kruskal BA, et al. Development and validation of an automated HIV prediction algorithm to identify candidates for pre-exposure prophylaxis: a modelling study. The Lancet HIV. 2019;6(10):e696-704.

79. Damen JA, Pajouheshnia R, Heus P, Moons KGM, Reitsma JB, Scholten RJPM, et al. Performance of the Framingham risk models and pooled cohort equations for predicting 10-year risk of cardiovascular disease: a systematic review and meta-analysis. BMC Med. 2019;17(1):109.
80. Berwick DM. Disseminating innovations in health care. JAMA : the journal of the American Medical Association. 2003;289(15): 1969-75.

81. Institute of M. Committee on Quality of Health Care in America. Crossing the quality chasm: a new health system for the 21 st century. National Academies Press. 2001.

82. Balas EA, Boren SA. Managing clinical knowledge for health care improvement. Yearb Med Inform. 2000;9(01):65-70.

83. Dopson S, Locock L, Chambers D, Gabbay J. Implementation of evidence-based medicine: evaluation of the promoting action on clinical effectiveness programme. J Health Serv Res Policy. 2001;6(1):23-31.

84. Malcolm N, Rollison J, Clarke Erickson, MHA, Hart J. Development of a decision-making tool to expand access to PrEP services for women. 2019.

85. Powell BJ, McMillen JC, Proctor EK, et al. A compilation of strategies for implementing clinical innovations in health and mental health. Med Care Res Rev. 2012;69(2):123-57.

86. Proctor EK, Powell BJ, McMillen JC. Implementation strategies: recommendations for specifying and reporting. Implement Sci. 2013;8(1):139.

87. Karris MY, Beekmann SE, Mehta SR, Anderson CM, Polgreen PM. Are we prepped for preexposure prophylaxis (PrEP)? Provider opinions on the real-world use of PrEP in the United States and Canada. Clin Infect Dis: An Official Publication of the Infectious Diseases Society of America. 2014;58(5):704-12.

88. White JM, Mimiaga MJ, Krakower DS, Mayer KH. Evolution of Massachusetts physician attitudes, knowledge, and experience regarding the use of antiretrovirals for HIV prevention. AIDS Patient Care STDs. 2012;26(7):395-405.

89. Tripathi A, Ogbuanu C, Monger M, Gibson JJ, Duffus WA. Preexposure prophylaxis for HIV infection: healthcare providers' knowledge, perception, and willingness to adopt future implementation in the southern US. South Med J. 2012;105(4):199-206.

90. Landovitz RJ. Preexposure prophylaxis for HIV prevention: what we know and what we still need to know for implementation. Top Antivir Med. 2015;23(2):85-90.

91. Coy KC, Hazen RJ, Kirkham HS, Delpino A, Siegler AJ. Persistence on HIV preexposure prophylaxis medication over a 2year period among a national sample of 7148 PrEP users, United States, 2015 to 2017. J Int AIDS Soc. 2019;22(2):e25252.

92. Siegler AJ, Komro KA, Wagenaar AC. Law everywhere: a causal framework for law and infectious disease. Public Health Rep. 2020;135(1 suppl):25S-31S

93. Weiss G, Smith DK, Newman S, Wiener J, Kitlas A, Hoover KW. PrEP implementation by local health departments in US cities and counties: findings from a 2015 assessment of local health departments. PLoS One. 2018;13(7):e0200338.

94. Antonisse L, Garfield R, Rudowitz R, Artiga S. The effects of Medicaid expansion under the ACA: updated findings from a literature review. In:2018.

95. Siegler AJ, Mehta CC, Mouhanna F, et al. Policy- and county-level associations with HIV pre-exposure prophylaxis use, the United States, 2018. Ann Epidemiol. 2020;45:24-31 e23.

96. Force UPST. Preexposure prophylaxis for the prevention of HIV infection: US preventive services task force recommendation statement. JAMA : the journal of the American Medical Association. 2019;321(22):2203-13

97. Hughes R, Rosacker N, Sloan C. Coverage of federally recommended preventive services: considerations for eliminating HIV transmission by 2030. Health Affairs. 2019 (2019).

Publisher's Note Springer Nature remains neutral with regard to jurisdictional claims in published maps and institutional affiliations. 